\title{
ENFOQUE DE UNA SALUD EN LAS ACCIONES PARA ENFRENTAR LA RESISTENCIA A LOS ANTIMICROBIANOS DESDE UNA ÓPTICA LATINOAMERICANA
}

\author{
Pilar Ramon Pardo ${ }^{1, a}$, Hatim Sati ${ }^{1, a}$, Marcelo Galas ${ }^{1, b}$
}

\begin{abstract}
RESUMEN
La resistencia a los antimicrobianos es un problema global de epidemiología compleja, adecuado para un enfoque amplio e integrado de «Una Salud». Existen organismos resistentes en humanos, animales, alimentos y el medio ambiente, y el principal impulsor de esta resistencia es el uso de antimicrobianos. La aparición y propagación de la resistencia a los antimicrobianos continúa sin cesar en todo el mundo, dejando devastadores resultados de salud y económicos a su paso. En el 2015, la OMS, en colaboración con socios clave como la FAO y la OIE, desarrolló el Plan de Acción Mundial en Resistencia a los Antimicrobianos, bajo la perspectiva de «Una Salud». Este Plan fue endosado por los países y busca la colaboración para la prevención y control de la resistencia a los antimicrobianos. En este artículo, se pretende explicar el alcance del enfoque «Una Salud» en el contexto de la resistencia a los antimicrobianos, por qué se ha adoptado y qué se espera lograr con ello. Una de las conclusiones principales es la gran falta de conocimiento sobre la selección y propagación de organismos resistentes en el medio ambiente, con énfasis en las amenazas y riesgos que esto representa para la salud humana y animal. Se han de intensificar los esfuerzos para mejor definir los riesgos, diseñar las intervenciones y medir su impacto en la resistencia a los antimicrobianos.
\end{abstract}

Palabras clave: Resistencia a los antimicrobianos, Latinoamérica, Vigilancia epidemiológica. (fuente: DeCS BIREME).

\section{"ONE HEALTH" APPROACH IN THE ACTIONS TO ADDRESS ANTIMICROBIAL RESISTANCE FROM A LATIN AMERICAN STANDPOINT}

\begin{abstract}
Antimicrobial resistance is a complex epidemiology problem worldwide which calls for a wide and integrated approach such as "One Health." Resistant organisms are present in humans, animals, food, and the environment, and the main impeller of this resistance is the use of antimicrobial agents. The occurrence and propagation of antimicrobial resistance continue incessantly in the world, leaving devastating economic and health consequences behind. In 2015, the WHO, in collaboration with key partners such as the FAO and the OIE, developed the World Action Plan in Antimicrobial Resistance, under the perspective of "One Health." This Plan was endorsed by several countries and seeks collaboration for the prevention and control of antimicrobial resistance. This article aims at explaining the scope of the "One Health" approach within the context of antimicrobial resistance, the reason behind its adoption and what is expected from it. One of the main conclusions is the significant lack of knowledge on the selection and propagation of resistant organisms in the environment, with an emphasis on the threats and risks that this represents to human and animal health. Efforts need to be strengthened in order to better define the risks, design interventions, and measure its impact on antimicrobial resistance.
\end{abstract}

Keywords: antimicrobial resistance; Latin America; Surveillance. (source: MeSH NLM).

Pan American Health Organization (PAHO). Washington, DC, EE. UU.

Médico cirujano; ${ }^{\mathrm{b}}$ Bioquímico

Recibido: 22/02/2018 Aprobado: 07/03/2018 En línea: 05/04/2018

Citar como: Ramon-Pardo P, Sati H, Galas M. Enfoque de una salud en las acciones para enfrentar la resistencia a los antimicrobianos desde una óptica latinoamericana. Rev Peru Med Exp Salud Publica. 2018;35(1):103-9. doi: 10.17843/rpmesp.2018.351.3605. 
"La salud humana y animal están ligadas de manera inextricable. Siempre ha sido así, y así siempre será».

James H Steele (1913 - 2013), Chief, Veterinary Public Health Division, CDC. Assistant Surgeon General for Veterinary Affairs, USPHS

\section{INTRODUCCIÓN}

La resistencia a los antimicrobianos se ha convertido en un problema político, social y económico de nuestro tiempo. La eficacia de los antimicrobianos está disminuyendo rápidamente, junto con la aparición de bacterias panresistentes y la diseminación incontrolada de genes resistentes a antibióticos en entornos no clínicos. Algunas proyecciones sugieren que en 2050 morirán más personas por infecciones causadas por bacterias resistentes que de cáncer ${ }^{(1)}$.

La resistencia a los antimicrobianos es un problema global de epidemiología compleja, adecuado para un enfoque amplio e integrado de «Una Salud». Existen organismos resistentes en humanos, animales, alimentos y el medio ambiente, y el principal impulsor de esta resistencia es el uso de antimicrobianos. La aparición y propagación de la resistencia a los antimicrobianos continúa sin cesar en todo el mundo, dejando devastadores resultados de salud y económicos a su paso. Esas consecuencias se multiplicarán si no se toman medidas globales de colaboración para abordar la propagación de la resistencia. Los principales impulsores de la resistencia a los antimicrobianos en los seres humanos se han acelerado por la prescripción inadecuada de antimicrobianos en las prácticas de atención de la salud; el uso inapropiado de antimicrobianos en el ganado; y la propagación de genes de resistencia a antibióticos en el medio ambiente.

La gran diversidad de Latinoamérica plantea muchos retos en relación con la vigilancia epidemiológica de las enfermedades en general; y de la resistencia a los antimicrobianos específicamente. La región abarca una amplia variedad de paisaje geográfico, clima y diversidad biológica ${ }^{(2)}$. Desde el punto de vista económico, los países latinoamericanos se clasifican en su mayoría como países de ingreso mediano bajo ${ }^{(3)}$, también se caracterizan por tener una de las mayores variaciones en cuanto a los recursos y estructura socioeconómica ${ }^{(4)}$. Aunque la región ha logrado un progreso social y económico constante, una gran proporción de la población continúa viviendo en la pobreza. La vivienda y el saneamiento inadecuados aceleran la propagación de los patógenos y genes resistentes de persona a persona y el medio ambiente.

El propósito de este artículo es profundizar en el significado del enfoque «Una Salud» en el contexto de la resistencia a los antimicrobianos, por qué se ha adoptado y qué se espera lograr con ello. Hacer frente a la creciente amenaza de la resistencia a los antimicrobianos requiere un enfoque holístico y multisectorial («Una Salud») porque los antimicrobianos utilizados para tratar diversas enfermedades infecciosas en animales pueden ser los mismos o similares a los utilizados en humanos. El enfoque de «Una Salud» también abarca el medio ambiente, otro vínculo adicional entre humanos y animales, y también una fuente potencial de nuevos microorganismos resistentes.

\section{UNA SALUD Y RESISTENCIA A LOS ANTIMICROBIANOS}

One Health o "Una Salud» es un término utilizado para describir un principio que reconoce que la salud humana y animal están interconectadas, que las enfermedades se transmiten de los humanos a los animales y viceversa y, por lo tanto, deben abordarse en desde ambos aspectos. Es un enfoque concebido para diseñar y aplicar programas, políticas, leyes e investigaciones en el que múltiples sectores se comunican y colaboran para lograr mejores resultados de salud pública. El enfoque de "Una Salud» es especialmente pertinente en la inocuidad de los alimentos, el control de zoonosis (como la gripe, la rabia, la fiebre amarilla, la cisticercosis, la leptospirosis, la peste, entre otras) y la resistencia a los antibióticos.

Las bacterias resistentes que se originan en humanos, animales $o$ el medio ambiente pueden propagarse de un país a otro. La resistencia a los antimicrobianos no reconoce fronteras geográficas o humano / animal. La Organización Mundial de la Salud (OMS), la Organización para la Agricultura y la Alimentación (FAO) y la Organización Mundial de Sanidad Animal (OIE) hablan con una sola voz y toman medidas colectivas para minimizar la aparición y propagación de la resistencia. Los objetivos son asegurar que los agentes antimicrobianos continúen siendo efectivos y útiles para curar enfermedades en humanos y animales; promover el uso prudente y responsable de los agentes antimicrobianos y asegurar el acceso global a medicamentos de buena calidad ${ }^{(5)}$.

La emergencia y diseminación de nuevos mecanismos de resistencia es un ejemplo de la importancia de «Una Salud». Así, en 2016, la identificación del nuevo gen mcr-1 que confiere la resistencia a colistina, motivó una alerta epidemiológica por parte de la OPS ${ }^{(6)}$. Un estudio retrospectivo de prevalencia del gen mcr-1 en aislamientos de $E$ coli y K. pneumoniae recolectados entre abril de 2011 y noviembre de 2014 en China demostró la presencia de bacterias $E$ coli portadoras del gen mcr-1 en 78 (15\%) de 523 muestras de carne cruda, 166 (21\%) de 804 muestras de animales y $16(1 \%)$ de 1322 muestras de pacientes hospitalizados con infección ${ }^{(7)}$. Posteriormente, otros países informaron sobre hallazgos similares realizados de manera retrospectiva. El gen mcr-1 fue detectado en base de datos y colecciones de cepas bacterianas en todos los continentes, también en Latinoamérica. Se aislaron muestras de bacterias, como E. coli y Salmonellas, portadoras de dicho gen procedentes de varias fuentes, tanto de humanos (aislamientos procedentes de la comunidad) como también en carnes de cerdos y aves ${ }^{(8)}$. Estos hallazgos corroboran 
la transferencia de genes, mediados por plásmidos, entre diferentes especies.

En la última década los enterococos se han convertido en una de las causas más importantes de infecciones asociadas a la atención de la salud. El linezolid, primer miembro de una nueva clase de antibióticos, llamada oxazolidinonas, aprobada en Estados Unidos para uso en humanos en el año 2000, ha sido introducido en la práctica clínica para su uso en infecciones causadas por bacterias grampositivas multirresistentes, especialmente para el enterococo resistente a vancomicina y para el Staphylococcus aureus resistente a la meticilina.

Recientemente se identificó un nuevo gen, designado como optrA, que confiere resistencia a las oxazolidinonas y a los fenicoles (tanto cloranfenicol como florfenicol) en Enterococo faecalis y Enterococo faecium, en muestras de origen humano y animal ${ }^{(9)}$. En cuanto al impacto en salud pública, estudios recientes ponen de manifiesto el hallazgo de aislamientos portadores de este nuevo mecanismo de resistencia en $E$. faecalis y $E$. faecium, tanto en muestras clínicas humanas, como en muestras de animales, principalmente de cerdos y pollos destinados al consumo. Aunque los datos de los que se dispone en estos estudios no proporcionan evidencia directa sobre la transmisión de patógenos optrA positivos entre animales y humanos, es importante destacar que los aislamientos de Enterococo faecalis de humanos y cerdos tenían un patrón similar por electroforesis en gel de campo pulsado (PFGE por sus siglas en inglés) y el mismo perfil analizado por secuenciación multilocus (MLST por sus siglas en inglés).

A pesar de estos hallazgos de mecanismos emergentes de resistencia, se requieren más evidencias y discusión sobre las implicancias y efectos en la salud humana del movimiento de genes de resistencia en diferentes ecosistemas.

Se han descrito numerosos elementos que pueden influir en la selección y diseminación de mecanismos emergentes de resistencia a los antimicrobianos que pueden abarcar: políticas regulatorias y técnicas de manejo de desechos con respecto al uso de antimicrobianos en la agricultura animal; el papel y la efectividad de los programas de uso optimizado de antibióticos para reducir y prevenir la resistencia a los antimicrobianos (en salud humana y animal); la disponibilidad e intercambio de datos para monitorear y evaluar la implementación y el progreso de las estrategias; el desarrollo de estrategias para mantener la efectividad de los medicamentos existentes, para desarrollar nuevos medicamentos y diagnósticos, y para implementar estrategias de prevención de enfermedades, incluido el uso de vacunas y las alternativas a los antibióticos; y la necesidad de mecanismos nacionales e internacionales de colaboración y coordinación en los dominios de «Una Salud» para la prevención y control, así como la investigación y el desarrollo ${ }^{(10)}$.
En Latinoamérica, se puede reconocer que la regulación de la producción y venta de antibióticos es débil, tanto en el sector humano como en la agricultura y sector veterinario. Los antibióticos están disponibles sin receta en muchos países de la región, lo que resulta en un uso indiscriminado ${ }^{(11)}$.

Además, la difusión generalizada de bacterias y genes resistentes se produce en el medio ambiente y los sistemas alimentarios. La contaminación coliforme del agua corriente todavía es un problema, en algunas áreas de Latinoamérica se encuentra entre las más altas del mundo, con genes resistentes a los antibióticos registrados en los sistemas de suministro de agua potable en varios países de la región. El uso indiscriminado de antibióticos en el ganado y la acuicultura para el tratamiento y la promoción del crecimiento ha acelerado la aparición y propagación de patógenos resistentes a los antibióticos transmitidos por los alimentos $^{(12)}$.

Uno de los principales desafíos a nivel global es la falta de regulación sobre la contaminación en la producción o manufactura de antimicrobianos. A pesar de que se proponen límites para la regulación medioambiental (13), estimados en función de las concentraciones inhibitorias mínimas de los antibióticos, todavía la metodología presenta desafíos por la competitividad entre las especies y genotipos. Es prioritario desarrollar líneas de investigación que: guíen los esfuerzos de la industria en la producción de antibióticos; y que permitan a las agencias reguladoras definir emisiones aceptables de antibióticos en el medio ambiente, tomando en cuenta los riesgos para la selección de microorganismos resistentes.

\section{IMPLICANCIAS Y RESPUESTA DESDE LAS POLIITICAS DE SALUD INTERNACIONALES}

En mayo de 2015, el Plan de Acción Mundial para la Resistencia a los Antimicrobianos fue adoptado por sexagésima octava Asamblea Mundial de la Salud, a través de la resolución WHA $68.7^{(14,15)}$. Este plan se elaboró con la colaboración de varios socios clave como la FAO y la OIE, bajo una óptica de colaboración en la prevención y contención de la resistencia a los antimicrobianos. El plan de acción mundial tiene por objeto "asegurar la continuidad, mientras sea posible, del tratamiento y la prevención satisfactorios de enfermedades infecciosas con medicamentos eficaces, seguros y de calidad garantizada, que se usen de modo responsable y sean accesibles a todas las personas que los necesiten". Este Plan se elaboró bajo la perspectiva de la salud, por lo que se subraya la importancia de replicar este enfoque en el ámbito nacional. Así, los planes nacionales de acción han de seguir las normas y directrices establecidas por organismos intergubernamentales como la Comisión del Codex Alimentarius, la FAO y la OIE. 
En la misma línea, los países de las Américas respaldaron el Plan de Acción Regional sobre Resistencia a los Antimicrobianos, adoptado por el 55to Consejo Directivo de la OPS ${ }^{(16)}$. Alineado con el Plan Mundial, el plan regional prioriza una estrategia coordinada hacia la mejora de la vigilancia integrada de la resistencia a los antimicrobianos, con una respuesta multisectorial al reconocer las diversas necesidades de salud pública, recursos y prácticas dentro de la región.

Tras el endose del Plan de Acción Mundial por la Asamblea Mundial de la Salud, se realizaron dos reuniones históricas de alto nivel: la sesión especial sobre resistencia a los antimicrobianos en la Asamblea General de las Naciones Unidas ${ }^{(17)}$ en 2016 y la declaración del G20 (Hamburgo, 2017) ${ }^{(18)}$, donde se confirmó el compromiso de los países para abordar la resistencia a los antimicrobianos desde una perspectiva multisectorial. Uno de los acuerdos más importantes tras la sesión de Naciones Unidas, fue la necesidad de un enfoque colaborativo amplio, que divulgue y socialice la discusión de la resistencia a los antimicrobianos más allá del entorno de la salud. Se conformó un Grupo Coordinador Interinstitucional sobre la Resistencia a los Antimicrobianos (IACG, por sus siglas en inglés), compuesto por los organismos de las Naciones Unidas pertinentes, otras asociaciones internacionales y expertos en el tema.

\section{ESTRATEGIAS DE RESPUESTA "UNA SALUD"}

\section{UNA RESPUESTA ARTICULADA DESDE LA VIGILANCIA}

La vigilancia de las enfermedades tiene como objetivo proporcionar datos e información para la toma de decisiones; este mismo principio se cumple respecto a la vigilancia de la resistencia a los antimicrobianos ${ }^{(19)}$. Esta vigilancia ha cobrado una gran relevancia en el contexto de los planes nacionales de acción, para el monitoreo y evaluación de su impacto. Otra función de la vigilancia es la detección y notificación de mecanismos emergentes de resistencia, que han de ser oportunamente informados a aquellos que tengan que tomar acción sobre los mismos (trabajadores de salud, responsables ministeriales, agencias nacionales e internacionales e investigadores).

El enfoque «Una Salud» es un marco conceptual óptimo para realizar la vigilancia de la resistencia, que permite la integración de los diferentes aspectos de la vigilancia, más allá de la salud humana. Algunos autores proponen un marco conceptual integrado ${ }^{(20}$, en el que se incluyen todos los aspectos relacionados con el consumo de antimicrobianos en humanos y animales, el medio ambiente, y el monitoreo de las resistencias en microorganismos aislados en alimentos, animales y seres humanos. Estos sistemas han de estar basados en técnicas de laboratorio estándares y gestión de los datos, el cual puede ser integrado, comparado e interpretado a todos los niveles.

En 1996, en alianza con países latinoamericanos, la OPS/OMS lanzó la primera red formal de vigilancia de la resistencia a los antimicrobianos en la región: la Red Latinoamericana para la Vigilancia de Resistencia a los Antimicrobianos (ReLAVRA). La meta es recopilar los datos de calidad de la resistencia a los antimicrobianos para mejorar el conocimiento sobre el tema y, en consecuencia, proporcionar la base para la toma de decisiones y políticas de salud pública pertinentes a la prevención y contención de la resistencia a los antimicrobianos. Hoy en día, la red sigue activa, como una de las iniciativas de vigilancia más antiguas a nivel mundial (19,21). Desde entonces, la OPS/ OMS y los países han ampliado y fortalecido los programas para la vigilancia de la resistencia a los antimicrobianos, gracias a las colaboraciones con diferentes socios técnicos y financieros.

En Latinoamérica, además de ReLAVRA, dedicada a la vigilancia de la resistencia en patógenos humanos, existen otras dos redes orientadas a la vigilancia de la resistencia en alimentos. En el 2004, los Centros para el Control y la Prevención de Enfermedades de los Estados Unidos (CDC) en colaboración con la OPS/OMS iniciaron un programa en América Latina, denominada red de vigilancia de Pulse Net Latinoamérica, con la meta de fortalecer la cooperación técnica entre países participantes. Está conformada por los laboratorios de referencia en 14 países (Argentina, Bolivia, Brasil, Chile, Colombia, Costa Rica, Cuba, Guatemala, México, Nicaragua, Paraguay, Perú, Uruguay y Venezuela) (22). El Instituto Nacional de Enfermedades Infecciosas (INEI) - ANLIS Carlos G. Malbrán, es el laboratorio de referencia designado, proporcionando el programa de control externo de la calidad para la red

La red ha desempeñado un papel importante, estableció la capacidad en los países participantes para la identificación del genotipo de cepas de bacterias patógenas con protocolos estandarizados. La vigilancia de redes se centra en agentes patógenos seleccionados (Salmonella spp., Vibrio cholerae, Escherichia coliO157, Shigella spp., Campylobacter spp., Listeria monocitogenes), creando una base de datos regional de los aislamientos ${ }^{(23)}$. En fecha muy reciente, la red ha estado explorando la posibilidad de completar la vigilancia con técnicas moleculares (Secuenciación Completa de Genoma) para informar decisiones clínicas y la caracterización genética de agentes patógenos que circulan en la región ${ }^{(24)}$. Este último tiene el potencial para mejorar la detección temprana y la interpretación de brotes anticipados y localmente en secuencia ${ }^{(25)}$.

La Red Mundial de Infecciones Transmitida por los Alimentos de la OMS (GFN, por sus siglas en inglés) constituye una red de instituciones e individuos lanzada en el 2011 por el Departamento de Inocuidad de los Alimentos, Zoonosis y Enfermedades de Transmisión Alimentaria de la OMS. Su 
meta es apoyar a los países para fortalecer la vigilancia nacional y regional integrada, la investigación, la prevención, la respuesta y el control de infecciones transmitidas por los alimentos y otras infecciones entéricas ${ }^{(26,27)}$. La red GFN es coordinada por el centro colaborador de la OPS/OMS, Instituto Nacional de Alimentación en Dinamarca, que también administra el sistema de garantía de la calidad externo para serotipificación y el antibiograma de agentes patógenos transmitidos por los alimentos ${ }^{(28)}$.

\section{PARAR EL USO DE ANTIBIÓTICOS EN ANIMALES SANOS}

En algunos países, aproximadamente el $80 \%$ del consumo total de antibióticos de importancia médica se produce en el sector animal, principalmente para estimular el crecimiento en animales sanos. Esto sin duda pone un riesgo en la selección y diseminación de las resistencias, que pueden transmitirse a los seres humanos a través de los alimentos o del medio ambiente. De manera consecuente, muchos países han implementado restricciones sobre el uso de antibióticos en la agricultura, en un esfuerzo por combatir el aumento de la resistencia a los antibióticos en los humanos.

El impacto de la restricción del uso de antibióticos en animales destinados a la producción de alimentos se ha podido evidenciar en la disminución de la presencia de bacterias resistentes en los animales, hasta en un $15 \%$, y la bacteria multirresistente entre un 24 y $32 \%{ }^{(29)}$. Las evidencias apuntan a que este efecto se podría mostrar también en seres humanos, con una reducción de hasta el $24 \%$ de bacterias resistentes, pero todavía se requieren más estudios para demostrar este efecto. Estos hallazgos han sustentado las directrices de la OMS sobre el uso de antimicrobianos de importancia médica en animales destinados a la producción de alimentos ${ }^{(30)}$.

Las nuevas recomendaciones de la OMS tienen como finalidad preservar la eficacia de los antibióticos importantes para la medicina humana reduciendo su uso innecesario en animales. La OMS recomienda firmemente una reducción general del uso de todas las clases de antibióticos de importancia médica en los animales destinados a la producción de alimentos, incluida la restricción completa de estos fármacos para estimular el crecimiento y prevenir enfermedades sin diagnóstico previo. Solo se debería administrar antibióticos a animales sanos para prevenir una enfermedad, en caso haya sido diagnosticada en otros animales de la misma cabaña o población de peces.

Muchos países ya han adoptado medidas para reducir el uso de antibióticos en animales destinados a la producción de alimentos. Por ejemplo, en 2006 la Unión Europea prohibió el uso de antibióticos para estimular el crecimiento ${ }^{(31)}$. Los consumidores también están impulsando la demanda de carne producida sin el uso sistemático de antibióticos, por lo que algunas cadenas alimentarias importantes están adoptando la política de "ausencia de antibióticos» para sus suministros cárnicos. El cambio de cultura de los consumidores es crítico para poder influenciar en las políticas y regulaciones nacionales. De manera inmediata, se han de promover opciones al uso de antibióticos para prevenir enfermedades en animales como la mejora de la higiene, un mejor uso de la vacunación, y la introducción de cambios en las prácticas de estabulación y cría de animales. Estas medidas suponen un cambio en las prácticas de los productores de alimentos que pueden requerir inversión adicional y apoyo por parte de las autoridades.

\section{LA LISTA DE ANTIMICROBIANOS DE IMPORTANCIA CRÍTICA PARA LA MEDICINA HUMANA: UNA HERRAMIENTA EFECTIVA PARA PRESERVAR LOS ANTIMICROBIANOS}

Para preservar el uso de antimicrobianos críticos para la salud humana y limitar su uso en veterinaria, la OMS publicó una Lista de antimicrobianos de importancia crítica para la medicina humana por primera vez en 2005. Su objetivo es promover el uso prudente de dichos fármacos y enlentecer el desarrollo de resistencias. En esta lista, que se actualiza periódicamente, se clasifican todos los antibióticos utilizados actualmente en humanos y animales en tres categorías - «importantes», «muy importantes»y «de importancia crítica»- en función de su relevancia para la medicina humana.

En la más reciente revisión de la lista (abril de 2017) ${ }^{(32)}$ los antibióticos considerados de máxima prioridad entre los antimicrobianos de importancia crítica son las quinolonas, las cefalosporinas de tercera generación o más, los macrólidos y ketólidos, los glicopéptidos y las polimixinas (también conocidas como colistinas). Estos antibióticos son esenciales como tratamientos de último recurso para infecciones multirresistentes en humanos, generalmente de origen hospitalario.

\section{CONCLUSIONES}

La resistencia a los antimicrobianos es un ejemplo que muestra que la salud humana está conectada a la de los animales y al medio ambiente; de manera que solo un esfuerzo multidisciplinario puede proporcionar una adecuada respuesta.

En este momento, existe una gran falta de conocimiento sobre la selección y propagación de organismos resistentes en el medio ambiente, con énfasis en las amenazas y riesgos que esto representa para la salud humana y animal. Por ejemplo, la presencia de antimicrobianos en el medio ambiente a través de los desechos por seres humanos, animales y fabricación. Se deben evaluar los procedimientos de desechos y se deben desarrollar nuevas tecnologías para permitir un uso eficiente y rápido, así como la degradación de antimicrobianos en plantas de tratamiento de aguas residuales, corrientes de desechos orgánicos o ambiente. 
Es necesario seguir estudiando la viabilidad y la implementación de programas de monitoreo y vigilancia, incluido el desarrollo de un monitoreo armonizado de antimicrobianos y microorganismos resistentes en el medio ambiente, en el contexto de la vigilancia integrada. El uso de datos armonizados de la vigilancia y de la investigación, permitirá desarrollar metodologías de evaluación de riesgos para la salud humana y animal. En el sector agroalimentario, los vínculos entre las prácticas agrícolas, la salud animal y el desarrollo y propagación de la resistencia a los antimicrobianos deben investigarse en profundidad.

Es preciso que organismos internacionales, nacionales y académicos apoyen la investigación de las brechas de conocimiento sobre la liberación de microorganismos resistentes y antimicrobianos en el medio ambiente y su propagación; explorar metodologías de evaluación de riesgos y utilizarlas para evaluar los riesgos para la salud humana y animal de la presencia de antimicrobianos en el medio ambiente; apoyar la investigación y el desarrollo de nuevas herramientas para el control de antimicrobianos y microorganismos resistentes en el medio ambiente; apoyar el desarrollo de tecnologías que permitan la degradación eficiente y rápida de antimicrobianos en aguas residuales y el medio ambiente y reducir la propagación de la resistencia a los antimicrobianos.

Contribuciones de autoría: PRP concibió la idea y redactó el artículo con la colaboración de HS. MG y NB revisaron críticamente el contenido e hicieron sugerencias al mismo.

Fuentes de financiamiento: Autofinanciado.

Conflictos de interés: Los autores declaran no tener conflictos de interés.

\section{REFERENCIAS BIBLIOGRÁFICAS}

1. Review on Antimicrobial Resistance. Tackling drug-resistant infections globally: final report and recommendations [internet]. London, (United Kingdom); 2016. [cited 2018 Jan 10]. Disponible en: https://amr-review.org/sites/default/ files/160525_Final\%20paper_with\%20 cover.pdf

2. Dangles O, Loirat J, Freour C, Serre S, Vacher J, Le Roux X. Research on biodiversity and climate change at a distance: collaboration networks between Europe and Latin America and the Caribbean. PLoS One. 2016;11(6):e0157441. doi: 10.1371/journal.pone.0157441.

3. World Bank Group (WBG). World Development Indicator Database. World Development Indicators Database, [Internet]. Washington DC: World Bank Group; 2017 [cited 2018 Jan 10]. Disponible en: https://data. worldbank.org/indicator/NY.GNP. PCAP.CD ?locations $=X M-X D-X T$ $\mathrm{XN}$ \&view=map

4. De Andrade LO, Pellegrini Filho A, Solar O, Rígoli F, de Salazar LM, Serrate PC, Ribeiro KG, Koller TS, Cruz FN, Atun R. Social determinants of health, universal health coverage, and sustainable development: case studies from Latin American countries. Lancet. 2015 4;385(9975):1343-51. doi: 10.1016/ S0140-6736(14)61494-X.

5. Food and Agriculture Organization of the United Nations, World Organisation for
Animal Health, World Health Organization. Antimicrobial resistance: a manual for developing national action plans. 2016. [cited 2018 Jan 10]. Disponible en: http:// apps.who.int/iris/ bitstream/10665/204 470/1/9789241549530_eng.pdf?ua=1

6. Organización Panamericana de la Salud / Organización Mundial de la Salud. Alerta Epidemiológica: Enterobacterias con resistencia transferible a colistina. Implicaciones para la salud publica en las Américas. 10 de junio de 2016, Washington, D.C.: OPS/OMS. 2016. [cited 2018 Jan 10]. Disponible en:http:// www.paho.org/hq/index.php?option $=$ com_docman $\&$ task $=$ doc_view $\&$ Itemid $=270 \&$ gid $=35009$ \&lang $=$ es

7. Liu YY, Wang Y, Walsh TR, Yi LX, Zhang R, Spencer J. et al. Emergence of plasmid-mediated colistin resistance mechanism mcr-1 in animals and human beings in China: a microbiological and molecular biological study. Lancet Infect Dis. 2016;16(2):161-8. doi: 10.1016/ S1473-3099(15)00424-7.

8. Scov RL and Monnet DL. Plasmid-mediated colistin resistance (mcr-1 gene): three months later, the story unfolds. Euro Surveill. 2016;21(9):30155. doi: 10.2807/1560-7917.ES.2016.21.9.30155.

9. He T, Shen Y,Schwarz S, CaiJ,Lv Y,LiJ.et al. Genetic environment of the tranferable oxazolidinone/phenicol resistance gene optrA in Enterococcus faecalis isolates of human and animal origin. J Antimicrob
Chemother. 2016 Jun;71(6):1466-73. doi: $10.1093 / \mathrm{jac} / \mathrm{dkw0} 016$.

10. National Academies of Science, Ingineering and Medicine. Combating Antimicrobial Resistance: A One Health Approach to a Global Threat: Proceedings of a Workshop. Ceci Mundaca-Shah, V. Ayano Ogawa, and Anna Nicholson, Rapporteurs. Forum on Microbial Threats. Board on Global Health - Health and Medicine Division. Disponible en http://www.nationalacademies.org/ hmd/Reports/2017/combating-antimicrobial-resistance-proceedings.aspx

11. Wirtz VJ, Herrera-Patino JJ, Santa-Ana-Tellez Y, Dreser A, Elseviers M, Vander Stichele RH. Analysing policy interventions to prohibit overthe-counter antibiotic sales in four Latin American countries. Trop Med Int Health. 2013;18(6):665-73. doi: 10.1111/tmi.12096.

12. Singh PK. One Health approach to tackle antimicrobial resistance in South East Asia. BMJ. 2017;358:j3625. doi: 10.1136/bmj.j3625.

13. Bengtsson-Palme J, Larsson J. Concentrations of antibiotics predicted to select for resistant bacteria: Proposed limits for environmental regulation. Environ Int. 2016;86:140-9. doi: 10.1016/j.envint.2015.10.015.

14. Resolution WHA. 68.7. Global action plan on antimicrobial action. Sixty-eighth World Health Assembly, Agenda item. 2015 May;15:26. 
15. World Health Organization. Global Action Plan on Antimicrobial resistance. Geneva, (Switzerland): WHO; 2015. [cited 2018 Jan 10]. Disponible en: http:// www.who.int/antimicrobial-resistance/ publications/global-action-plan/en/

16. Pan American Health Organization. 54th Directing Council - 67th Session of the Regional Committee of WHO for the Americas. Washington, D.C., USA, 28 September-2 October 2015. Disponible en: file://C:/Users/satihat/Downloads/ CD54-12-e\%20(1).pdf

17. Naciones Unidas. Political declaration of the high-level meeting of the General Assembly on antimicrobial resistance [internet]. 71st Session of the United Nations General Assembly; 2016 Oct 5; New York: 2016 (Resolution A/RES/71/3) [cited 2018 Jan 10]. Disponible en: http://www.who.int/antimicrobial-resistance/interagency-coordination-group/ UNGA-AMR-RES-71-3-N1631065. pdf ?ua $=1$

18. G20 Germany-Hamburg Leaders' Declaration Shaping an Interconnected World. https://www.b20germany.org/fileadmin/user_upload/documents/B20/ B20_G20_Leaders_Declaration_Evaluation.pdf

19. Grundmann H, Klugman KP, Walsh T, Ramon-Pardo P, Sigauque B, Khan W, Laxminarayan R, Heddini A, Stelling J. A framework for global surveillance of antibiotic resistance. Drug Resist Updat. 2011;14(2):79-87. doi: 10.1016/j. drup.2011.02.007.

20. Queenan K, Häsler B, Rushton J, One Health approach to antimicrobial resistance surveillance: is there a business case for it?. Int J Antimicrob Agents. 2016;48(4):422-7. doi: 10.1016/j.jiantimicag.2016.06.014

21. World Health Organization. Antimicrobial resistance: global report on surveillance. World Health Organization; 2014.

22. PulsNet International, The International Molecular Subtyping Network for Food Disease Surveillance, CDC, PulseNet
Latin America \& Carribean [Internet] cited 2018 Jan 10]. Available from:http:// www.pulsenetinternational.org/ networks/latinamerica/

23. Ribot EM, Hise KB. Future challenges for tracking foodborne diseases: PulseNet, a 20-year-old US surveillance system for foodborne diseases, is expanding both globally and technologically. EMBO Rep. 2016 Nov;17(11):1499-1505.

24. Nadon C, Van Walle I, Gerner-Smidt P, Campos J, Chinen I, ConcepcionAcevedoJ, Gilpin B,Smith AM,Kam KM, Perez E, Trees E. PulseNet International: Vision for the implementation of whole genome sequencing (WGS) for global food-borne disease surveillance. Euro Surveill. 2017 8;22(23). pii: 30544. doi: 10.2807/1560-7917

25. Baker KS, Campos J, Pichel M, Della Gaspera A, Duarte-Martínez F, Campos-Chacón E, Bolaños-Acuña HM, Guzman-Verri C, Mather AE, Velasco $\mathrm{SD}$, Rojas MZ. Whole genome sequencing of Shigella sonnei through PulseNet Latin America and Caribbean: advancing global surveillance of foodborne illnesses. Clin Microbiol Infect. 2017;23(11):845853. doi: 10.1016/j.cmi.2017.03.021.

26. World Health Organization (WHO). Global Foodborne Infections Network (GFN) [Internet]. Geneva: World Health Organization; 2017 [cited 2018 Jan 10]. Available from: http://www. who.int/gfn/en/

27. Hald T, Aspinall W, Devleesschauwer B, Cooke R, Corrigan T, Havelaar AH, Gibb HJ, Torgerson PR, Kirk MD, Angulo FJ, Lake RJ. World Health Organization estimates of the relative contributions of food to the burden of disease due to selected foodborne hazards: a structured expert elicitation. PLoS One. 2016;19;11(1):e0145839. doi: 10.1371/journal.pone.0145839.

28. Hendriksen RS, Vieira AR, Karlsmose S, Lo Fo Wong DM, Jensen AB, Wegener HC, Aarestrup FM. Global monitoring of Salmonella serovar distribution from the World Health Organization
Global Foodborne Infections Network Country Data Bank: results of quality assured laboratories from 2001 to 2007. Foodborne Pathog Dis. 2011;8(8):887900. doi: 10.1089/fpd.2010.0787

29. Tang KL, Caffrey NP, Nobrega DB, Cork SC, Ronksley PE, Barkema $\mathrm{HW}$, et al. Restricting the use of antibiotics in food-producing animals and its associations with antibiotic resistance in food-producing animals and human beings: a systematic review and meta-analysis. Lancet Planet Health. 2017;1(8):e316-e327. doi: $10.1016 /$ S2542-5196(17)30141-9.

30. Organización Mundial de la Salud. WHO guidelines on use of medically important antimicrobials in food-producing animals. Geneva: World Health Organization; 2017. Licence: CC BY-NCSA 3.0 IGO.OMS. [cited 2018 Jan 10]. Disponible en:, http://apps.who.int/iris/ bitstream/10665/258970/1/97892415 50130-eng.pdf ?ua $=1$

31. European Union Commission (2005): Ban on antibiotics as growth promoters in animal feed enters into effect.

Regulation 1831/2003/EC on additives for use in animal nutrition, replacing Directive $70 / 524 /$ EEC on additives in feed-stuffs, Brussels, 22 December. [cited 2018 Jan 10]. Disponible en: http:// europa.eu/rapid/press-release_IP-051687_en.htm

32. Organización Mundial de la Salud. Critically important antimicrobials for human medicine - 5th rev. Geneva: World Health Organization; 2017. License: CC BY-NC-SA 3.0 IGO [cited 2018 Jan 10]. Disponible en: http://apps.who.int/iris/ bitstream/10665/255027/1/97892415 12220-eng.pdf?ua $=1$

Correspondencia: Pilar Ramon Pardo

Dirección: 525, 23rd Street, NW, Washington DC. EUA

Teléfono: +12022307160

Correo electrónico: ramonpap@paho.org 